\title{
BMJ Open Mapping the evidence of experiences related to adolescent idiopathic scoliosis: a scoping review protocol
}

\author{
Maciej Płaszewski (D) , Weronika Grantham, Ejgil Jespersen
}

To cite: Płaszewski M, Grantham W, Jespersen E. Mapping the evidence of experiences related to adolescent idiopathic scoliosis: a scoping review protocol. BMJ Open 2019;9:e032865. doi:10.1136/ bmjopen-2019-032865

- Prepublication history and additional material for this paper are available online. To view these files, please visit the journal online (http://dx.doi. org/10.1136/bmjopen-2019032865).

Received 09 July 2019 Revised 14 October 2019 Accepted 30 0ctober 2019

Check for updates

(C) Author(s) (or their employer(s)) 2019. Re-use permitted under CC BY-NC. No commercial re-use. See rights and permissions. Published by BMJ.

Faculty of Physical Education and Health in Biała Podlaska, Józef Piłsudski University of Physical Education, Warsaw, Poland

Correspondence to Maciej Płaszewski; plaszewski@wp.pl

\begin{abstract}
Introduction Adolescent idiopathic scoliosis, the diagnosis and management of this condition, may lead to poorer body image and diminished psychosocial functioning. Furthermore, treatment, especially bracing and surgery as well as screening, remain controversial and debated, with an unclear evidence base. Personal experiences in terms of issues such as personcentred care, shared decision making, and patient and public involvement, are contemporarily recognised as highly valued. Nonetheless, people's experiences related to adolescent idiopathic scoliosis is an issue underrepresented in current systematic reviews and systematically developed recommendations. There
\end{abstract} appears a substantial imbalance between a vast amount of biomedical research reports, and sporadic biopsychosocial publications in this field. The objective of this planned scoping review is to explore and map the available evidence from various sources to address a broad question of what is known about experiences of all those touched, directly and indirectly, by the problem of adolescent idiopathic scoliosis.

Methods and analysis We based our protocol on the Joanna Briggs Institute's scoping review method, including the Population - Concept - Context framework, to formulate the objectives, research questions, eligibility criteria and conduct characteristics of the study. We will consider any primary study designs, research synthesis reports, as well as narrative reviews and opinion pieces. We will not restrict eligible publications to English language. Search and selection processes will include academic and grey literature searches using multiple electronic databases, search engines and websites, hand searches, and contacting the authors. We will use a customised data charting table and present a narrative synthesis of the results.

Ethics and dissemination Scoping review is a secondary study, aiming at synthesising data from publicly available publications, hence it does not require ethical approval. We will submit the report to a peer-reviewed journal and disseminate it among professionals involved in scoliosis management, guideline and recommendation development, and policymaking.

\section{INTRODUCTION}

Adolescent idiopathic scoliosis (AIS) is a complex health condition that is defined as a lateral spine curvature of $10^{\circ}$ or more, of

\section{Strengths and limitations of this study}

This article outlines a protocol of the first research synthesis study focusing on people's experiences related to adolescent idiopathic scoliosis, an issue underrepresented in current systematic reviews and systematically developed recommendations in this field.

- The scoping review characteristics, multiple database and hand searches for academic and grey literature, will increase the likelihood of thorough mapping of the evidence concerning this personcentred subject matter.

- We will use the Joanna Briggs Institute's Problem - Content - Context framework for the selection and analysis of the literature, and study report formulation.

- In addition to standard requirements for scoping review studies, to increase the trustworthiness of our findings, we will conduct critical appraisals of the included publications.

- For methodological and practical reasons, we will not consider sources from social networks and blogs, which is a potential limitation, given the subject matter of our study.

an unknown origin, that manifests in children older than 10 years of age. ${ }^{1-3}$ Mild AIS is present in about $1.5 \%-3 \%$ of adolescents, while more severe curves exceeding $40^{\circ}$ are found in $0.04 \%-0.3 \%$. The female to male ratio ranges from about 1.4:1 for curves of less than $20^{\circ}$ to $7.2: 1$ for curves exceeding $40^{\circ}$.

This structural deformity of the spine and trunk, depending on its severity, may lead to pain and pulmonary or cardiac complications. $^{1-4}$ On the other hand, this health condition, but potentially also the diagnosis and treatment, may be associated with lower self-esteem and poorer body image, as well as worse psychosocial functioning. ${ }^{15-7}$ All these may also touch significant others. ${ }^{89}$ Treatment of AIS, especially bracing and surgery, are controversial as regards side effects and harms, with inconsistent evidence base. ${ }^{15610-14}$ Even diagnostic imaging methods, and minimal 
spine and trunk asymmetry and deformity criteria, as well as cut-off points for the diagnosis of the condition, are under discussion in this context. ${ }^{13-15}$ Routine screening for scoliosis is also debated, with conflicting recommendations. ${ }^{16-18}$ The evidence base for both screening and treatment is very unclear. ${ }^{14151719}$

Based on recent comprehensive systematic reviews, ${ }^{13}{ }^{14}$ impactful narrative reviews, ${ }^{1-3}$ and tertiary evidence synthesis studies, ${ }^{17} 19$ little is considered and understood about what the people diagnosed with, and treated for, AIS, their significant others, and other people, experience about this condition. Furthermore, there appears a substantial imbalance between a vast amount of biomedical research reports, and sporadic biopsychosocial publications. ${ }^{14} 1719$ It is especially significant as this health problem emerges in a fragile time of puberty and adolescence and as ethical doubts have been raised concerning management of AIS. ${ }^{6} 1318$ The recommendations for research and management of $\mathrm{AIS}^{20-23}$ seem to uphold this state of affairs.

This is striking in the evidence-based practice perspective, since recommendation formulation principles have evolved in recent years. ${ }^{24-26}$ Experiences of people, in terms of issues such as person-centred care, shared decision making, and patient and public involvement, are contemporarily recognised as principal and highly valued. ${ }^{27-29}$ The evidence-based practice triad addresses expertise of professionals, evidence for effectiveness and safety of interventions, and a person's perspectives, with their opinions, attitudes, values and views. ${ }^{30}$ Those perspectives are also important in terms of the acceptance of treatment, an issue discussed in scoliosis management as being crucial and problematic. ${ }^{16}{ }^{19}$ More generally, personal factors concerning illness as a perceived, personal experience, in contrast to disease as a medical term, ${ }^{24283032}$ are vital as regards management of AIS. A better understanding of these aspects needs to be opened with mapping of evidence.

\section{Why scoping review}

To the best of our knowledge, research syntheses addressing various aspects of AIS, typically apply the standard method of systematic review of intervention studies, and are based exclusively on the evidence from controlled trials and quantitative observational studies. ${ }^{13} 141719$ Furthermore, none of the reviews included grey literature as sources of evidence. Consequently, potential reports of people's experiences were possibly excluded from those systematic reviews based primarily on study design selection criteria.

Therefore, the scoping review research synthesis method is warranted for our investigation. Scoping reviews 'serve a different purpose' than systematic reviews ${ }^{33}$ and are used to examine the presence, extent, variety and characteristics of the evidence. They are essentially exploratory and are not restricted to a focused research question and specific populations, interventions (exposures) and outcomes. ${ }^{33-35}$
This is a protocol of a scoping review with an evidence map. In the absence of available mapping of the volume and content of literature regarding people's experiences regarding AIS, an evidence map study is also warranted. ${ }^{36}$ Scoping studies are appropriate at initial stages of evidence mapping to identify knowledge gaps. ${ }^{33-35}$ Both research synthesis methods 'share similarities' with regards to methodology and reporting guidelines. ${ }^{33} 36$ Preferred Reporting Items for Systematic Reviews and Meta-Analyses (PRISMA) Extension for Scoping Reviews (PRISMA-ScR) applies both for scoping reviews and for evidence maps. ${ }^{34}$

\section{Objectives}

We are interested in people's experiences, defined as both 'something that happens to you that affects how you feel' (the passive mode) and 'the process of getting knowledge or skill from doing, seeing, or feeling things' (the active mode),${ }^{37}$ related to AIS. In terms of evidence-based practice $^{30-32}$ our aim is to map the evidence addressing people's experiences in terms of their perspectives, preferences, needs and values. This scoping review is not intended to address the term 'experience' understood as a component of expertise of professionals delivering treatment and care, gained through the years of training and routine.

The objectives of this scoping review are:

- To map and examine the extent, variety and nature of the evidence addressing experiences related to AIS.

- To explore the depth and the comprehensiveness of current understandings of people's experiences of AIS in everyday life and health and care contexts.

- To identify knowledge gaps in this subject matter.

Hence, the main question of the study is: what is known from the available reports about experiences of all those touched by the problem of AIS, both directly and indirectly-taking into consideration both the natural history and the untreated AIS, and the management of this health condition.

\section{METHODS AND ANALYSIS}

\section{Protocol design and reporting}

We based our protocol on the Joanna Briggs Institute's (JBI) scoping review manual ${ }^{35}$ and consulted the PRISMA-ScR checklist and explanation paper. ${ }^{34}$ Additionally, we referred to both the original Arksey and O'Malley's scoping review framework, ${ }^{38}$ and the methodological input from Levac et al. ${ }^{39}$ For the reporting of the protocol, we followed the JBI guidance, ${ }^{35}$ and consulted PRISMA-ScR ${ }^{3440}$ as well as PRISMA for protocols (PRISMA-P) ${ }^{41}$

\section{Eligibility criteria}

We adopted the JBI's Population - Concept - Context (PCC) framework $^{35}$ to formulate the objectives and research questions, and also to conceptualise the study and report characteristics in terms of eligibility criteria. 
Table 1 Objectives and eligibility criteria for the review

\begin{tabular}{|c|c|}
\hline Objectives/inclusion criteria & Elaboration \\
\hline $\begin{array}{l}\text { Population/ types of participants: } \\
\text { People with AIS } \\
\text { Their significant others } \\
\text { Other people involved }\end{array}$ & $\begin{array}{l}\text { People diagnosed with AIS, regardless of their age } \\
\text { Significant others: for example, parents, siblings, friends and } \\
\text { professionals in some cases } \\
\text { People involved in the management of AIS } \\
\text { Sources that exclusively focus on other than AIS types of scoliosis } \\
\text { (eg, scoliosis related to other health conditions, early onset scoliosis) } \\
\text { will not be considered }\end{array}$ \\
\hline $\begin{array}{l}\text { Concept/ phenomena of interest: } \\
\text { People's experience related to AIS } \\
\text { Size and volume/depth and breadth/ } \\
\text { comprehensiveness of the body of literature } \\
\text { regarding people's experience related to AIS }\end{array}$ & $\begin{array}{l}\text { Information sources regarding quality of life, body image, mood, } \\
\text { depression, anxiety, mental health, activities of daily living, and other } \\
\text { medical and social issues will be considered for inclusion if provide } \\
\text { experience-related body of evidence }\end{array}$ \\
\hline $\begin{array}{l}\text { Context/ setting: } \\
\text { - Everyday life } \\
\text { Healthcare context }\end{array}$ & $\begin{array}{l}\text { Country and culture: any country, regardless of cultural context (eg, } \\
\text { the issue of school screening is a subject of analyses in countries and } \\
\text { cultures worldwide) }\end{array}$ \\
\hline
\end{tabular}

AIS, adolescent idiopathic scoliosis.

The PCG characteristics of our study are elaborated on in table 1 .

\section{Eligible study designs}

We will consider any quantitative, qualitative and mixedmethods primary study designs, including different qualitative research methods like narrative, phenomenology, grounded theory, ethnography and case study, as well as any research synthesis reports. Narrative reviews and opinion pieces, including editorials, letters, debate, commentary and viewpoint papers, will also be considered. Publications such as essays, diaries, newspaper articles, newsletters, blogs, fiction, will not be considered as eligible. We will provide a list of excluded studies and publications, with reasons for exclusion.

\section{Other limits}

Sources in English, Polish, Scandinavian and German languages will be considered for inclusion. If found relevant (based on abstract, summary, table of contents, heading or introduction), for studies in Russian, French and Chinese, we will consider inviting colleagues with relevant expertise for collaboration as interpreters. There will be no restriction as to publication date but in the charting process sources will be analysed as relevant to current practice or as historical, based on their publication date, content and context. Commercial information and information provided by sources having potential conflicts of interests (eg, personal stories published on websites popularising diagnostic or treatment methods) will be excluded. We will not conduct searches of social networks and blogs. We will consider research papers concerning social media use addressing the objectives of our study.

\section{Information sources}

Given the subject matter, the characteristics of published relevant narrative reviews, ${ }^{1-3} 57{ }^{19}$ evidence synthesis reports, ${ }^{131419}$ research recommendations ${ }^{142023}$ and based on our preliminary searches, we assume that a search and selection process, including both academic and grey literature sources, is necessary for this study.

When deciding whether to qualify information sources as grey literature, we will follow the widely accepted 'Luxembourg definition' of grey literature as work that 'is produced on all levels of government, academia, business, and industry in print and electronic formats, but which is not controlled by commercial publishers, i.e. where publishing is not the primary activity of the producing body'. ${ }^{42}$ We are also informed by the explanation of grey literature complementing the AMSTAR 2 tool for quality appraisal of systematic reviews. ${ }^{43}$

\section{Search strategy}

We organised our exploratory search process into the following stages: (1) Academic literature search. (2) Grey literature search. (3) Complementary hand searches (snowballing searching) of the reference lists of the included publications. (4) Contacting authors.

\section{Electronic bibliographic databases}

To achieve satisfactory and required comprehensiveness and completeness of our searches, we need to conduct our searches both in a manner typical for scoliosis review studies-in medically oriented databases, and also in databases covering social sciences. We will search in general bibliographic and research synthesis databases. We will also search in databases provided by academic publishers as, especially for social science publications, we need to conduct searches in particular journals. Databases to be searched include PubMed, MEDLINE (via EBSCO), SportDiscus (via EBSCO), Web of Science including Social Sciences Citation Index, Scopus, ProQuest, PsycINFO, Social Sciences Full Text (EBSCO), JSTOR, GoogleScholar, Cochrane CENTRAL, JBI, Campbell Library, 
Epistemonikos, SpringerLink, ScienceDirect (Elsevier), Wiley Online Library and Taylor \& Francis Online. The list may be extended by including other key publishing houses.

\section{Grey literature}

We formulated the following grey literature search strategy: ${ }^{43} 44$

1. Grey literature databases search: Open Grey, Proquest Dissertations \& Thesis Global, New York Academy of Medicine's Grey Literature Report, Google Scholar, Web of Science.

2. The Grey Matters checklist.

3. Google search; we will search the first 10 pages for the search hits (ie, 100 records to be screened for each set of search terms), as a recommended method allowing to capture the most relevant records while maintaining feasibility.

4. Targeted web-based searches: websites of institutions, organisations and patient groups.

We will conduct the grey literature search, after concluding the academic literature search, so that we will be better acquainted with the search and selection process.

\section{Hand searching}

Hand searching of the reference lists of the included publications will be done consecutively throughout the searching process.

\section{Contacting the authors}

We will contact key authors known to publish in this area for any additional published or unpublished work.

We will conduct the searches using all identified keywords and index terms. The initial search strategies for PubMed and for grey literature, including a list of selected websites, are presented as online supplementary file 1 .

\section{Selection of sources, critical appraisal and data charting Organisation of the process}

The study team consists of two senior researchers, one of them with an expertise in scoliosis studies and in research synthesis methods, and one with expertise in phenomenology and in qualitative studies. The third author is a doctoral candidate with a background in phenomenology and in qualitative studies.

We will apply the iterative team approach to selecting sources, for data charting from and critical appraisal of the included literature. The screening and study selection, as well as data charting and critical appraisals will be undertaken by one reviewer and two verifiers, working independently. Then the reviewer and the verifiers will resolve discrepancies, if present, by discussion. If needed, we will invite two collaborators to support us with the data charting process.

\section{Selection of sources}

We will use two combined flow diagrams for academic and for grey literature search and selection processes, based directly on the PRISMA flow chart ${ }^{45}$ and adapted from Godin et $a l,{ }^{42}$ respectively. The results of both searches will be combined, and then, if applicable, supplemented with the results of the hand searches of the reference lists. We will use a hand search table for reporting results of hand searches. The template flow diagrams and a template hand search table are attached as online supplementary file 2.

\section{Data charting framework}

We will use a data charting table for the process of data charting from the included sources. Our aim is to use the form at the review stage, and we assume that the data charting process is iterative so that the charting table might be updated during the review process. The data charting form is attached as online supplementary file 3 .

\section{Methodological quality appraisal}

During the data charting process, we will additionally assess methodological quality of the included literature. We will use the Mixed Methods Appraisal Tool, V.2018, ${ }^{46}$ which is designed to appraise the methodological quality of empirical studies-qualitative research, randomised controlled trials, non-randomised studies, quantitative descriptive studies and mixed-methods studies, as well as the JBI Checklist for Text and Opinion ${ }^{47}$ for theoretical or opinion publications. For systematic reviews of randomised or non-randomised studies of interventions, we will apply the AMSTAR $2^{43}$ tool.

\section{Calibration exercises}

We will conduct pilot tests (calibration exercises) to ensure systematic and reproducible study selection, and to confirm satisfactory inter-rater agreements, as well as to familiarise the review team with the data charting form and to test the comprehensiveness of its content. The data charting form will be trialled on two reports. ${ }^{712}$

\section{Characteristics of the included sources of evidence}

We will present characteristics for which data were charted and will provide the citations for each source of evidence in an evidence summary table, ${ }^{34-36}$ corresponding to the data charting table. It will be presented in the final report to map the evidence regarding the objectives of this scoping review.

\section{Protocol registration}

We made our protocol publicly visible via the Open Science Framework (OSF) website (https://osf.io/ 3yr76/, created 07 February 2019).

\section{Changes to the protocol}

Given the exploratory characteristics of the study, we can expect amendments to the search and selection process, and, consequently, to the data charting table during the review process. If done, this will be reported through the OSF registry and in the final report. 


\section{Key dates}

We made our first attempts to this scoping review starting in November 2018, and conducted initial exploratory searches in February 2019. We expect to start the actual study in November 2019 and to prepare the report by July 2020.

\section{Patient and public involvement}

There was no patient or public involvement in the creation of this protocol and is not planned in the review, in accordance with the objectives of this study.

\section{DISCUSSION}

The management of AIS needs to be considered, in terms of person-centred aspects of care, including people's experiences and everyday life beyond health professional settings. This scoping review is intended to supplement the body of evidence with a research synthesis report regarding people's experiences regarding AIS. Implementation of the wide and exploratory scoping review research synthesis method, rather than a systematic review approach, is ideal for that purpose.

The exploratory and open characteristics of the scoping review approach, both as regards methodology, and the subject matter of this study, allows us to conduct the review in an iterative, evolving way. Nonetheless, we faced some important issues at the stage of creating the protocol.

\section{Conduct guidelines considerations}

We chose the current JBI guidance ${ }^{35}$ for scoping review conduct, as it is consistent with the PRISMA-ScR guidance, ${ }^{34}$ and it addresses, uses and improves earlier scoping review methodology proposals. ${ }^{36} 38$ More importantly, the JBI model of evidence-based healthcare ${ }^{32}$ corresponds with the concept of our study, with the principles of the evidence for feasibility, appropriateness, meaningfulness of interventions for specific populations, cultures and contexts, as being of equal value to the evidence of effectiveness. This model acknowledges the broad conceptualisation of evidence, with the pronunciation of varying sources of evidence.

\section{Reporting guidelines considerations}

We will follow the PRISMA-ScR reporting guidelines in the final report of the scoping review. As to the protocol reporting, PRISMA-P is the standard reporting guideline for systematic review protocols, ${ }^{41}$ while the only general guidance for the content of scoping review protocols is provided in the JBI manual. ${ }^{35}$ Therefore, in order to specify the most accurate checklist and content of our protocol, we conducted a comparative exercise of the PRISMA-ScR (in two slightly differing versions ${ }^{34} 40$ ) and the corresponding items of the PRISMA-P.

\section{Application of results}

This scoping review is intended to identify existing practice and research gaps to inform researchers, and all those involved in the management and care of people diagnosed with AIS, including practitioners, policymakers, and interest groups and organisations in the field. Especially, this scoping review can inform developers of recommendations and practice guidelines.

\section{Strengths and limitations considerations}

Methodological quality, risk of bias and strength of evidence

Our goal is to map the available publications, with minimal restrictions as to study designs, and with a wide grey literature search, in order to identify evidence gaps and research needs. Critical appraisal of the methodological quality or risk of bias within the individual sources of evidence is not expected for scoping reviews ${ }^{33} 3438$ Nonetheless, to strengthen the trustworthiness of our study, we will conduct a critical appraisal of the included individual publications. We are not going to conduct any syntheses of results or any assessment of the overall strength of the body of evidence.

Social media and blogs as sources of evidence. Despite the potential large body of knowledge attainable from those sources, taking into account methodology guidance for scoping studies, ${ }^{35363839}$ and probable difficulties in meaningful and sound analyses of such texts, ${ }^{48} 49$ we assumed that including social media analyses is inapplicable within this review. A separate study is probably required for that task.

The planned recipients of the included sources of evidence (eg, be it a peer-reviewed journal report produced as a scientific activity or a solicited report for a stakeholder, such as agency or a committee, or other policymaker), as well as study author affiliations (eg, whether the authors are independent or connected to a scoliosis treatment clinic or to a spinal deformity scientific organisation or a group of professionals), are potential important factors in relation to the characteristics of the included sources of evidence, and their trustworthiness. We will describe those characteristics, as well as sources of funding for the included sources of evidence, in a text and in a separate table.

\section{Evidence mapping}

The graphical representation of evidence mapping in the final report will be done as tabular qualitative summaries and flow diagrams of the searching and selection processes, as well as tables containing characteristics of hand search results and characteristics of relevant websites and online materials, but not bubble plots. This is consistent with the characteristics and requirements for evidence maps. $^{36}$

\section{Consultations}

The optional, sixth stage of the scoping review framework (Stage 6: Consultation), as originally proposed by Arksey and O'Malley, ${ }^{38}$ involves consultations with key stakeholders in order to broaden the literature searching and selection process (ie, to include further sources of knowledge indicated by the stakeholders) and to receive 
their feedback as to the findings of the scoping review. Our scoping review is, however, not intended to involve consultation with stakeholders for translating knowledge at this stage of the study. Our aim is to examine and to synthesise the body of literature, and to distribute the findings. The implementation and dissemination stage is too distant at this point.

Contributors MP contributed with the idea of the review and proposed the design of the work. MP, EJ and WG conceptualised the study and MP and WG implemented the scoping review frameworks. MP drafted and edited the manuscript and the supplementary material, and EJ and WG revised it critically and contributed for its final version. All authors read and approved the final version of the manuscript.

Funding The authors have not declared a specific grant for this research from any funding agency in the public, commercial or not-for-profit sectors.

Competing interests None declared.

Patient consent for publication Not required.

Provenance and peer review Not commissioned; externally peer reviewed.

Open access This is an open access article distributed in accordance with the Creative Commons Attribution Non Commercial (CC BY-NC 4.0) license, which permits others to distribute, remix, adapt, build upon this work non-commercially, and license their derivative works on different terms, provided the original work is properly cited, appropriate credit is given, any changes made indicated, and the use is non-commercial. See: http://creativecommons.org/licenses/by-nc/4.0/.

ORCID iD

Maciej Płaszewski http://orcid.org/0000-0001-9874-5964

\section{REFERENCES}

1 Weinstein SL, Dolan LA, Cheng JCY, et al. Adolescent idiopathic scoliosis. The Lancet 2008;371:1527-37.

2 Altaf F, Gibson A, Dannawi Z, et al. Adolescent idiopathic scoliosis. BMJ 2013;346:f2508.

3 Hresko MT. Idiopathic scoliosis in adolescents. N Engl J Med Overseas Ed 2013:368:834-41.

4 Konieczny MR, Senyurt H, Krauspe R. Epidemiology of adolescent idiopathic scoliosis. J Child Orthop 2013;7:3-9.

5 Tones M, Moss N, Polly DW. A review of quality of life and psychosocial issues in scoliosis. Spine 2006;31:3027-38.

6 Linker B. A dangerous curve: the role of history in America's scoliosis screening programs. Am J Public Health 2012;102:606-16.

7 Carrasco MIB, Ruiz MCS, Bonilla Carrasco MI. [Perceived selfimage in adolescent idiopathic scoliosis: an integrative review of the literature]. Rev Esc Enferm USP 2014;48:748-57.

8 Du C, Yu J, Zhang J, et al. Relevant areas of functioning in patients with adolescent idiopathic scoliosis on the International classification of functioning, disability and health: the patients' perspective. $J$ Rehabil Med 2016;48:806-14

9 Wang H, Li T, Yuan W, et al. Mental health of patients with adolescent idiopathic scoliosis and their parents in China: a cross-sectional survey. BMC Psychiatry 2019;19:147.

10 Weinstein SL, Dolan LA, Wright JG, et al. Effects of bracing in adolescents with idiopathic scoliosis. $N$ Engl J Med 2013;369:1512-21.

11 Schwieger T, Campo S, Weinstein SL, et al. Body image and qualityof-life in untreated versus Brace-Treated females with adolescent idiopathic scoliosis. Spine 2016;41:311-9.

12 Rullander A-C, Isberg S, Karling M, et al. Adolescents' experience with scoliosis surgery: a qualitative study. Pain Manag Nurs 2013;14:50-9.

13 NSC. Screening for adolescent idiopathic scoliosis: external review against programme appraisal criteria for the UK national screening Committee (UK NSC). London: UK national screening Committee Bazian Ltd; 2015.

14 Dunn J, Henrikson NB, Morrison CC, et al. Screening for adolescent idiopathic scoliosis: a systematic evidence review for the U.S. preventive services Task force. Rockville, MD: Agency for Healthcare Research and Quality, 2018: 319. 173.

15 Clark EM, Tobias JH, Fairbank J. The impact of small spinal curves in adolescents who have not presented to secondary care: a population-based cohort study. Spine 2016;41:E611-7.
16 Sarwark JF, Davis MM. Evolving recommendations for scoliosis screening: a compelling need for further research. JAMA 2018;319:127-9.

17 Płaszewski M, Bettany-Saltikov J. Are current scoliosis school screening recommendations evidence-based and up to date? A best evidence synthesis umbrella review. Eur Spine J 2014;23:2572-85.

18 Kuroki H, Department of Orthopaedic Surgery, National Hospital Organization Miyazaki Higashi Hospital, 4374-1 Tayoshi Ooaza Miyazaki, Japan. School Scoliosis Screening - World Trends and the Problems Encountered in Japan. Int J Orthop 2017;4:814-8.

19 Płaszewski M, Bettany-Saltikov J. Non-Surgical interventions for adolescents with idiopathic scoliosis: an overview of systematic reviews. PLoS One 2014;9:e110254.

20 Beauséjour M, Goulet L, Parent S, et al. The effectiveness of scoliosis screening programs: methods for systematic review and expert panel recommendations formulation. Scoliosis 2013;8:12.

21 Horne JP, Flannery R, Usman S. Adolescent idiopathic scoliosis: diagnosis and management. Am Fam Physician 2014;89:193-8.

22 Negrini S, Dincer F, Kiekens C, et al. Evidence based position paper on physical and rehabilitation medicine (PRM) practice for people with spinal deformities during growth. The European PRM position (UEMS PRM section). Eur J Phys Rehabil Med 2017;53:125-31.

23 Negrini S, Hresko TM, O'Brien JP, et al. Recommendations for research studies on treatment of idiopathic scoliosis: consensus 2014 between SOSORT and SRS non-operative management Committee. Scoliosis 2015;10:8.

24 Harris R, Sawaya GF, Moyer VA, et al. Reconsidering the criteria for evaluating proposed screening programs: reflections from 4 current and former members of the U.S. preventive services Task force. Epidemiol Rev 2011;33:20-35.

25 Andermann A, Blancquaert I, Beauchamp S, et al. Revisiting Wilson and Jungner in the genomic age: a review of screening criteria over the past 40 years. Bull World Health Organ 2008;86:317-9.

26 Brodersen J, Kramer BS, Macdonald H, et al. Focusing on overdiagnosis as a driver of too much medicine. BMJ 2018;362.

27 Ghanouni A, Renzi C, Meisel SF, et al. Common methods of measuring 'informed choice' in screening participation: Challenges and future directions. Prev Med Rep 2016;4:601-7.

28 Bombard Y, Baker GR, Orlando E, et al. Engaging patients to improve quality of care: a systematic review. Implement Sci 2018;13.

29 Stacey D, Légaré F, Lewis K, et al. Decision AIDS for people facing health treatment or screening decisions. Cochrane Database Syst Rev 2017;19.

30 Guyatt $\mathrm{G}$, Tonelli $\mathrm{M}$. The role of experience in an evidence-based practice. Med Roundtable Gen Med Ed 2012;1:75-84.

31 Greenhalgh T, Snow R, Ryan S, et al. Six 'biases' against patients and carers in evidence-based medicine. BMC Med 2015;13:200.

32 Jordan Z, Lockwood C, Aromataris E, et al. The updated JBI model for evidence-based healthcare. The Joanna Briggs Institute, 2016.

33 Tricco AC, Zarin W, Ghassemi M, et al. Same family, different species: methodological conduct and quality varies according to purpose for five types of knowledge synthesis. J Clin Epidemiol 2018;96:133-42.

34 Tricco AC, Lillie E, Zarin W, et al. PRISMA extension for scoping reviews (PRISMA-ScR): checklist and explanation. Ann Intern Med 2018;169:467-73.

35 Peters MDJ, Godfrey C, Mclnerney P. Scoping Reviews. In: Aromataris E, Munn Z, eds. Joanna Briggs Institute reviewer's manual. The Joanna Briggs Institute, 2017. https://reviewersmanual. joannabriggs.org/

36 Miake-Lye IM, Hempel S, Shanman R, et al. What is an evidence MAP? A systematic review of published evidence maps and their definitions, methods, and products. Syst Rev 2016;5:28

37 'experience'. Cambridge Online Dictionary. Available: https:// dictionary.cambridge.org/dictionary/english/experience [Accessed 1 Jul 2019].

38 Arksey H, O'Malley L. Scoping studies: towards a methodological framework. Int J Soc Res Methodol 2005;8:19-32.

39 Levac D, Colquhoun H, O'Brien KK. Scoping studies: advancing the methodology. Implementation Sci 2010;5.

40 Tricco AC, Lillie A, Zarin W, et al. Preferred reporting items for systematic reviews and meta-analyses extension for scoping reviews (PRISMA-ScR) checklist. Available: http://www.equator-network.org/ reporting-guidelines/prisma-scr/ [Accessed 1 Jul 2019].

41 Moher D, Shamseer L, Clarke M, et al. Preferred reporting items for systematic review and meta-analysis protocols (PRISMA-P) 2015 statement. Syst Rev 2015;4:1.

42 Godin K, Stapleton J, Kirkpatrick SI, et al. Applying systematic review search methods to the grey literature: a case study examining guidelines for school-based breakfast programs in Canada. Syst Rev 2015;4:138. 
43 Shea BJ, Reeves BC, Wells G, et al. AMSTAR 2: a critical appraisal tool for systematic reviews that include randomised or nonrandomised studies of healthcare interventions, or both. BMJ 2017;358.

44 Stapleton J, Fuller K, Lenton E. How to Find \& Document Grey Literature. Toronto. Canada: University of Waterloo, University of Toronto, 2018Available:. https:// guides. library. utoronto. ca/ c. php? $g=577919 \& p=4123572$

45 Moher D, Liberati A, Tetzlaff J, et al. Preferred reporting items for systematic reviews and meta-analyses: the PRISMA statement. BMJ 2009;339:b2535.
46 Hong QN, Pluye P, FbreguesS, et al. Mixed methods appraisal tool (MMAT), version 2018. Industry Canada: Canadian Intellectual Property Office, 2018.

47 The Joanna Briggs Institute critical appraisal tools for use in JBI systematic reviews checklist for text and opinion, 2017. Available: http://joannabriggs.org/research/critical-appraisal-tools.html

$48 \mathrm{Ng}$ JP, Tarazi N, Byrne DP, et al. Scoliosis and the social media: Facebook as a means of information exchange. Spine Deform 2017;5:102-8.

49 Kjellberg S, Haider J, Sundin O. Researchers' use of social network sites: a scoping review. Libr Inf Sci Res 2016;38:224-34. 